\title{
A comparison of two least-squared random coefficient autoregressive models: with and without autocorrelated errors
}

\author{
Autcha Araveeporn \\ Department of Statistics, King Mongkut's Institute of Technology Ladkrabang, Thailand \\ E-mail:kaautcha@hotmail.com
}

\begin{abstract}
This paper compares a Least-Squared Random Coefficient Autoregressive (RCA) model with a Least-Squared RCA model based on Autocorrelated Errors (RCA-AR). We looked at only the first order models, denoted RCA(1) and RCA(1)-AR(1). The efficiency of the Least-Squared method was checked by applying the models to Brownian motion and Wiener process, and the efficiency followed closely the asymptotic properties of a normal distribution. In a simulation study, we compared the performance of $\mathrm{RCA}(1)$ and $\mathrm{RCA}(1)-\mathrm{AR}(1)$ by using the Mean Square Errors (MSE) as a criterion. The RCA(1) exhibited good power estimation in both cases where the data is stationary and nonstationary. On the other hand, when data oscillates around its mean, RCA(1)-AR(1) performed better. For real world data, we applied the two models to the daily volume of the Thai gold price and found that $\mathrm{RCA}(1)-\mathrm{AR}(1)$ performed better than $\mathrm{RCA}(1)$.
\end{abstract}

Keywords: Autocorrelated Errors, Brownian motion, Mean Square Errors, Random Coefficient Autoregressive, Wiener process

\section{Introduction}

In recent years, models of time series data have been applied to the fields of finance, business, and economy. Time series data in the field of economy can either show a stationary or a nonstationary time series data. There are several stationary models fitted the stationary data such as the Autoregrssive (AR) model, Moving Average (MA) model, and Autoregressive Moving Average (ARMA) model.

For nonstationary time series data, the Autoregressive Integrated Moving Average (ARIMA) model can be used. The RCA Random Coefficient Autoregressive (RCA) model, introduced by Nicholls and Quinn [1], is another one. Presently, it is very popular because it uses past data to help estimate parameters.

Nicholls and Quinn [1] employed the least square method and the maximum likelihood method to estimate parameters. Wang and Ghosh [2] used the Bayesian approach to obtain the first order estimate of an RCA model. Thavaneswaran and Abraham [3] applied an estimating function to nonlinear time series data. The estimating function technique is equivalent to a weighted least square estimator discussed by Hwang and Basawa [4] and Chandra and Taniguchi [5].

Prediction based on an estimate of a time series model is difficult because of the effect of autocorrelated error. To address this type of error, various nonlinear autoregressive models have shown up in the literature: Haggan and Ozaki [6] modeled nonlinear vibration by using an amplitude-dependent autoregressive time series model entitled Exponential Autoregressive (EXPAR) model; Tong [7] introduced the Threshold Autoregressive (TAR) model of nonlinear time series; and Chan and Tong [8] developed a TAR model into the Smooth-Transition Autoregressive (STAR) model.

This study investigated an RCA model based on autocorrelated error and proposed applying the Least Square (LS) method to estimate its parameters as well as compared it to a model without autocorrelated error. The efficiency of an LS estimator can be validated by applying it to Brownian motion and Wiener process. Finally, 
we compared the performance of the LS method with that of the Mean Square Error (MSE) method using both simulated and real data.

The rest of the paper is organized as follows: Section 2 describes two RCA Models, with and without autocorrelated error; Section 3 describes the LS method for parameter estimation; Section 4 showed the efficiency of the LS estimator. We applied our proposed method to simulated data and real data (Thai daily gold price) in Section 5 and 6. Finally, in Section 7, we discussed the results and indicated some directions for further research.

\section{The RCA model with autocorrelated errors}

The general class of Random Coefficient Autoregressive model of order p, Wang and Ghosh [2] written RCA(p) by

$$
\begin{aligned}
& x_{t}=\alpha+\sum_{i=1}^{p} \beta_{t i} x_{t-i}+\varepsilon_{t} \quad, t=1,2, \ldots, n, \\
& \underline{\beta}_{t}=\underline{\mu}_{\beta}+\Sigma_{\beta}^{1 / 2} \underline{u}_{t},
\end{aligned}
$$

where $\alpha$ is the scalar of constant, $\underline{\beta}_{t}=\left(\beta_{t 1}, \ldots, \beta_{t p}\right)^{\top}$ are the sequence of iid (independent and identically distributed) random variables, $\underline{\mu}_{\beta}=\left(\mu_{\beta 1}, \ldots, \mu_{\beta p}\right)^{\top}$ and $\varepsilon_{t}$ 's are the iid from a distribution with mean zero and unit variance. Empirically, we can see that the time series data are appeared the autocorrelated in error terms which is applied by AutoRegressive (AR) process as follows:

$$
\varepsilon_{t}=\sum_{j=1}^{q} \rho_{j} \varepsilon_{t-j}+e_{t} \quad, t=1,2, \ldots, n .
$$

The RCA model with autocorrelated errors denoted $\mathrm{RCA}(\mathrm{p})-\mathrm{AR}(\mathrm{q})$ can be rewritten by

$$
\begin{aligned}
& x_{t}=\alpha+\sum_{i=1}^{p} \beta_{t i} x_{t-i}+\sum_{j=1}^{q} \rho_{j} \varepsilon_{t-j}+e_{t} \quad, t=1,2, \ldots, n, \\
& \underline{\beta}_{t}=\underline{\mu}_{\beta}+\Sigma_{\beta}^{1 / 2} \underline{u}_{t} .
\end{aligned}
$$

where $\underline{\rho}=\left(\rho_{1}, \ldots, \rho_{q}\right)^{\top}$ is the $q \times 1$ vector of constant.

In this paper, we will study the first order of RCA model with autocorrelated errors denoted by $\mathrm{RCA}(1)-\mathrm{AR}(1)$ that can be written as

$$
\begin{aligned}
x_{t} & =\alpha+\beta_{t} x_{t-1}+\rho \varepsilon_{t-1}+e_{t} \quad, t=1,2, \ldots, n, \\
\beta_{t} & =\mu_{\beta}+\sigma_{\beta} u_{t} .
\end{aligned}
$$

where $x_{t}$ 's are iid random variables with mean $\mu_{\beta}$, and variance $\sigma_{\beta}^{2}, \varepsilon_{t}$ 's are iid random variables with mean 0 and variance $\sigma_{\varepsilon}^{2}$, and $\beta_{t}$ 's and $\varepsilon_{t}$ 's are independent.

For the parameter estimation of $\mathrm{RCA}(1)-\mathrm{AR}(1)$, it can be seen from (4) that consisted of the intercept term $\alpha$, the mean $\mu_{\beta}$, variance $\sigma_{\beta}^{2}$ of the coefficient $\beta_{t}$, the variance $\sigma_{\varepsilon}^{2}$ of the $\varepsilon_{t}$, the coefficient $\rho$ of AR process, and the variance $\sigma_{e}^{2}$ of the $e_{t}$, or defined as $\theta=\left(\alpha, \mu_{\beta}, \sigma_{\beta}^{2}, \sigma_{\varepsilon}^{2}, \sigma_{e}^{2}, \rho\right)^{\top}$.

\section{Parameter estimation for RCA(1)-AR(1)}

To estimate parameter of $\mathrm{RCA}(1)-\mathrm{AR}(1)$, we propose the concept of LS method to estimate parameter $\theta=$ $\left(\alpha, \mu_{\beta}, \sigma_{\beta}^{2}, \sigma_{\varepsilon}^{2}, \sigma_{e}^{2}, \rho\right)^{\top}$ by minimizing sum of residuals square in 3 steps.

The first step, we consider following $\mathrm{RCA}(1)$ model

$$
\begin{aligned}
x_{t} & =\alpha+\beta_{t} x_{t-1}+\varepsilon_{t} \quad, t=1,2, \ldots, n, \\
\beta_{t} & =\mu_{\beta}+\sigma_{\beta} u_{t} .
\end{aligned}
$$

The parameter of $\operatorname{RCA}(1)$ is $\left(\mu_{\beta}, \sigma_{\beta}^{2}, \sigma_{\varepsilon}^{2}\right)$ based on the LS estimation. Given the sample $x_{1}, x_{2}, \ldots, x_{n}$, let $\varepsilon_{t}=$ $x_{t}-\alpha-\mu_{\beta} x_{t-1}$, then $\hat{\alpha}$ is to estimate by minimizing sum of square errors,

$$
\varepsilon_{t}=x_{t}-\alpha-\mu_{\beta} x_{t-1}
$$




$$
\begin{aligned}
\sum_{t=1}^{n}\left(\varepsilon_{t}\right)^{2} & =\sum_{t=1}^{n}\left(x_{t}-\alpha-\mu_{\beta} x_{t-1}\right)^{2} \\
\frac{\partial}{\partial \alpha} \sum_{t=1}^{n}\left(\varepsilon_{t}\right)^{2} & =-2 \sum_{t=1}^{n}\left(x_{t}-\alpha-\mu_{\beta} x_{t-1}\right)=0 \\
\hat{\alpha} & =\frac{\sum_{t=1}^{n} x_{t}}{n}-\mu_{\beta} \frac{\sum_{t=1}^{n} x_{t-1}}{n} .
\end{aligned}
$$

The LS estimate of $\hat{\mu}_{\beta}$ is given by

$$
\begin{aligned}
\frac{\partial}{\partial \mu_{\beta}} \sum_{t=1}^{n}\left(\varepsilon_{t}\right)^{2} & =-2 \sum_{t=1}^{n}\left(x_{t}-\alpha-\mu_{\beta} x_{t-1}\right) x_{t-1}=0 \\
\hat{\mu}_{\beta} & =\frac{\sum_{t=1}^{n} x_{t} x_{t-1}-\hat{\alpha} \sum_{t=1}^{n} x_{t-1}}{\sum_{t=1}^{n} x_{t-1}^{2}}
\end{aligned}
$$

From (7), let us replace in (6) and the solution of $\hat{\alpha}$ is

$$
\hat{\alpha}=\frac{\sum_{t=1}^{n} x_{t-1}^{2} \sum_{t=1}^{n} x_{t}-\sum_{t=1}^{n} x_{t} x_{t-1} \sum_{t=1}^{n} x_{t-1}}{n \sum_{t=1}^{n} x_{t-1}^{2}-\left(\sum_{t=1}^{n} x_{t-1}\right)^{2}} .
$$

Thus $\hat{\mu}_{\beta}$ is computed by

$$
\hat{\mu}_{\beta}=\frac{n \sum_{t=1}^{n} x_{t} x_{t-1}-\sum_{t=1}^{n} x_{t} \sum_{t=1}^{n} x_{t-1}}{n \sum_{t=1}^{n} x_{t-1}^{2}-\left(\sum_{t=1}^{n} x_{t-1}\right)^{2}} .
$$

For RCA(1) model, it can be written as

$$
x_{t}=\hat{\alpha}+\hat{\mu}_{\beta} x_{t-1} \quad, t=1,2, \ldots, n .
$$

Hence, the estimated errors can be denoted by

$$
\hat{\varepsilon}_{t}=x_{t}-\hat{\alpha}+\hat{\mu}_{\beta} x_{t-1} \quad, t=1,2, \ldots, n .
$$

The second step, we assume that the errors of time series data have an autocorrelation function. The RCA(1)-AR(1) model used the concept of LS method by minimizing sum of square of autocorrelated errors as

$$
\begin{aligned}
e_{t} & =x_{t}-\hat{\alpha}-\hat{\mu}_{\beta} x_{t-1}-\rho \hat{\varepsilon}_{t-1} \quad, t=1,2, \ldots, n, \\
\sum_{t=1}^{n} e_{t} & =\sum_{t=1}^{n}\left(x_{t}-\hat{\alpha}-\hat{\mu}_{\beta} x_{t-1}-\rho \hat{\varepsilon}_{t-1}\right)^{2}, \\
\frac{\partial}{\partial \rho} \sum_{t=1}^{n}\left(e_{t}\right)^{2} & =-2 \sum_{t=1}^{n}\left(x_{t}-\hat{\alpha}-\hat{\mu}_{\beta} x_{t-1}+\rho \hat{\varepsilon}_{t-1}\right) \hat{\varepsilon}_{t-1}=0 .
\end{aligned}
$$

We get $\hat{\rho}$ by computing

$$
\hat{\rho}=\frac{\sum_{t=1}^{n} x_{t} \hat{\varepsilon}_{t-1}-\hat{\alpha} \sum_{t=1}^{n} \hat{\varepsilon}_{t-1}-\hat{\mu}_{\beta} \sum_{t=1}^{n} x_{t-1} \hat{\varepsilon}_{t-1}}{\sum_{t=1}^{n} \hat{\varepsilon}_{t-1}^{2}} .
$$

For $\mathrm{RCA}(1)-\mathrm{AR}(1)$ model, we can write

$$
x_{t}=\hat{\alpha}+\hat{\mu}_{\beta} x_{t-1}+\hat{\rho} \hat{\varepsilon}_{t-1} \quad, t=1,2, \ldots, n .
$$

The final step, we let $F_{t}$ be the information set up to time $t$, and denote $\varepsilon_{t}=x_{t}-\alpha+\mu_{\beta} x_{t-1}$, then it is seen that $E\left(\varepsilon_{t} \mid F_{t}\right)=0$, and $E\left(\varepsilon_{t}^{2} \mid F_{t}\right)=\sigma_{\varepsilon}^{2}+\sigma_{\beta}^{2} x_{t-1}^{2}$. The estimation process is to the form of residuals $\hat{\varepsilon}_{t}=x_{t}-\hat{\alpha}-\hat{\mu}_{\beta} x_{t-1}$, and let $r_{t}^{2}=\hat{\varepsilon}_{t}^{2}-\sigma_{\varepsilon}^{2}-\sigma_{\beta}^{2} x_{t-1}^{2}$. Nicholls and Quinn [1] showed the LS estimators of $\sigma_{\varepsilon}^{2}$ and $\sigma_{\beta}^{2}$ by regressing $\hat{\varepsilon}_{t}^{2}$ on $x_{t-1}^{2}$, which are equivalent to minimizing $\sum_{t=1}^{n}\left(\hat{\varepsilon}_{t}^{2}-\sigma_{\varepsilon}^{2}-\sigma_{\beta}^{2} x_{t-1}^{2}\right)^{2}$ given by

$$
\sum_{t=1}^{n} r_{t}^{2}=\sum_{t=1}^{n}\left(\hat{\varepsilon}_{t}^{2}-\sigma_{\varepsilon}^{2}-\sigma_{\beta}^{2} x_{t-1}^{2}\right)^{2}
$$


Consequently, the LS estimates of $\sigma_{\varepsilon}^{2}$ and $\sigma_{\beta}^{2}$ are obtained by

$$
\begin{aligned}
\frac{\partial}{\partial \sigma_{\varepsilon}^{2}} \sum_{t=1}^{n} r_{t}^{2} & =-2 \sum_{t=1}^{n}\left(\hat{\varepsilon}_{t}^{2}-\sigma_{\varepsilon}^{2}-\sigma_{\beta}^{2} x_{t-1}^{2}\right)=0 \\
\sum_{t=1}^{n} \hat{\varepsilon}_{t}^{2} & =n \sigma_{\varepsilon}^{2}+\sigma_{\beta}^{2} \sum_{t=1}^{n} x_{t-1}^{2} \\
\hat{\sigma}_{\varepsilon}^{2} & =\frac{\sum_{t=1}^{n} \hat{\varepsilon}_{t}^{2}-\sigma_{\beta}^{2} \sum_{t=1}^{n} x_{t-1}^{2}}{n}
\end{aligned}
$$

and

$$
\begin{aligned}
\frac{\partial}{\partial \sigma_{\beta}^{2}} \sum_{t=1}^{n} r_{t}^{2} & =-2 \sum_{t=1}^{n}\left(\hat{\varepsilon}_{t}^{2}-\sigma_{\varepsilon}^{2}-\sigma_{\beta}^{2} x_{t-1}^{2}\right) x_{t-1}^{2}=0 \\
\sum_{t=1}^{n} \hat{\varepsilon}_{t}^{2} x_{t-1}^{2} & =\sigma_{\varepsilon}^{2} \sum_{t=1}^{n} x_{t-1}^{2}+\sigma_{\beta}^{2} \sum_{t=1}^{n} x_{t-1}^{4} \\
\hat{\sigma}_{\beta}^{2} & =\frac{\sum_{t=1}^{n} \hat{\varepsilon}_{t}^{2} x_{t-1}^{2}-\sigma_{\varepsilon}^{2} \sum_{t=1}^{n} x_{t-1}^{2}}{\sum_{t=1}^{n} x_{t-1}^{4}}
\end{aligned}
$$

The $\hat{\sigma}_{\varepsilon}^{2}$ and $\hat{\sigma}_{\beta}^{2}$ can be written in general form as

$$
\begin{aligned}
\hat{\sigma}_{\varepsilon}^{2} & =\frac{\sum_{t=1}^{n} \hat{\varepsilon}_{t}^{2} \sum_{t=1}^{n} x_{t-1}^{4}-\sum_{t=1}^{n} x_{t-1}^{2} \sum_{t=1}^{n} \hat{\varepsilon}_{t}^{2} x_{t-1}^{2}}{n \sum_{t=1}^{n} x_{t-1}^{4}-\left(\sum_{t=1}^{n} x_{t-1}^{2}\right)^{2}}, \\
\hat{\sigma}_{\beta}^{2} & =\frac{n \sum_{t=1}^{n} \hat{\varepsilon}_{t}^{2} x_{t-1}^{2}-\sum_{t=1}^{n} \hat{\varepsilon}_{t}^{2} \sum_{t=1}^{n} x_{t-1}^{2}}{n \sum_{t=1}^{n} x_{t-1}^{4}-\left(\sum_{t=1}^{n} x_{t-1}^{2}\right)^{2}} .
\end{aligned}
$$

Notice that for $\sigma_{e}^{2}$, we let $F_{t}$ be the information set up to time $t$, and denote $e_{t}=x_{t}-\alpha+\mu_{\beta} x_{t-1}+\rho \varepsilon_{t-1}$, then it is seen that $E\left(e_{t} \mid F_{t}\right)=0$, and $E\left(e_{t}^{2} \mid F_{t}\right)=\sigma_{\varepsilon}^{2}+\sigma_{\beta}^{2} x_{t-1}^{2}+\sigma_{e}^{2} \rho^{2}$. The estimation of $\sigma_{e}^{2}$ is used by corresponding LS method as $\sigma_{\varepsilon}^{2}$, and $\sigma_{\beta}^{2}$. Let $\hat{e}_{t}=x_{t}-\hat{\alpha}-\hat{\mu}_{\beta} x_{t-1}-\hat{\rho}, s_{t}^{2}=\hat{e}_{t}^{2}-\sigma_{\varepsilon}^{2}-\sigma_{\beta}^{2} x_{t-1}^{2}-\hat{\sigma}_{e}^{2} \hat{\rho}^{2}$, and regress $\hat{e}_{t}^{2}$ on $\rho^{2}$, which are equivalent to minimizing $\sum_{t=1}^{n}\left(\hat{e}_{t}^{2}-\sigma_{\varepsilon}^{2}-\sigma_{\beta}^{2} x_{t-1}^{2}-\sigma_{e}^{2} \hat{\rho}^{2}\right)$, that

$$
\sum_{t=1}^{n} s_{t}^{2}=\sum_{t=1}^{n}\left(\hat{e}_{t}^{2}-\sigma_{\varepsilon}^{2}-\sigma_{\beta}^{2} x_{t-1}^{2}-\hat{\sigma}_{e}^{2} \hat{\rho}^{2}\right)^{2} .
$$

Using the concept of LS method, $\sigma_{e}^{2}$ is computed by

$$
\begin{aligned}
\frac{\partial}{\partial \sigma_{e}^{2}} \sum_{t=1}^{n} s_{t}^{2} & =-2 \sum_{t=1}^{n}\left(\hat{e}_{t}^{2}-\hat{\sigma}_{\varepsilon}^{2}-\hat{\sigma}_{\beta}^{2} x_{t-1}^{2}-\hat{\sigma}_{e}^{2} \hat{\rho}^{2}\right) \hat{\rho}^{2}=0 \\
\sum_{t=1}^{n} \hat{e}_{t}^{2} & =n \hat{\sigma}_{\varepsilon}^{2}+\hat{\sigma}_{\beta}^{2} \sum_{t=1}^{n} x_{t-1}^{2}-n \hat{\sigma}_{e}^{2} \hat{\rho}^{2} \\
\hat{\sigma}_{e}^{2} & =\frac{\sum_{t=1}^{n} \hat{e}_{t}^{2}-n \hat{\sigma}_{\varepsilon}^{2}-\hat{\sigma}_{\beta}^{2} \sum_{t=1}^{n} x_{t-1}^{2}}{n \hat{\rho}^{2}}
\end{aligned}
$$

\section{Efficiency of LS estimator}

\subsection{RCA(1) model}

The $\mathrm{RCA}(1)$ model is presented by

$$
x_{t}=\alpha+\mu_{\beta} x_{t-1}+\varepsilon_{t}, t=1,2, \ldots, n .
$$

The $\mathrm{RCA}(1)$ at (21) can be written in terms of the matrix form following a regression model

$$
\mathbf{Y}=\mathbf{X} \underline{\beta}+\underline{\varepsilon},
$$


where

$$
\mathbf{Y}=\left[\begin{array}{c}
x_{1} \\
\vdots \\
x_{n}
\end{array}\right] \quad, \quad \mathbf{X}=\left[\begin{array}{cc}
1 & x_{0} \\
\vdots & \vdots \\
1 & x_{n-1}
\end{array}\right] \quad, \quad \underline{\beta}=\left[\begin{array}{c}
\alpha \\
\mu_{\beta}
\end{array}\right] \text {, and } \underline{\varepsilon}=\left[\begin{array}{c}
\varepsilon_{1} \\
\vdots \\
\varepsilon_{n}
\end{array}\right]
$$

Nicholls and Quinn [1] showed that under the normality of $\beta$ and $\underline{\varepsilon}$, if the process is second order stationary, then $\hat{\theta}$ is consistent for $\theta$ and moreover, if the forth moments of $\beta$ and $\underline{\varepsilon}$, then $\sqrt{n}(\hat{\theta}-\theta)$ has a limiting normal distribution. In this case, it is shown that if the $\alpha$ and $\mu_{\beta}$ consistency estimates for $\hat{\alpha}$ and $\hat{\mu}_{\beta}$, then $\sqrt{n}(\hat{\alpha}-\alpha)$ and $\sqrt{n}\left(\hat{\mu}_{\beta}-\mu_{\beta}\right)$ has a limiting normal distribution.

Phillips [9] showed that the sample moment of $\left\{x_{t}\right\}$ converges to random functions of Brownian motion or Wiener process:

$$
\begin{array}{ccc}
n^{-3 / 2} \sum_{t=1}^{n} x_{t-1} & \stackrel{d}{\rightarrow} & \sigma_{\varepsilon} \int_{0}^{1} W(r) d r, \\
n^{-2} \sum_{t=1}^{n} x_{t-1}^{2} & \stackrel{d}{\rightarrow} & \sigma_{\varepsilon}^{2} \int_{0}^{1} W(r)^{2} d r, \\
n^{-1} \sum_{t=1}^{n} x_{t-1} \varepsilon_{t} & \stackrel{d}{\rightarrow} & \sigma_{\varepsilon}^{2} \int_{0}^{1} W(r) d W(r),
\end{array}
$$

where $W(r)$ denotes a standard Brownian motion. Using the above equation Phillips [9] showed that

$$
\begin{array}{rll}
n(\hat{\alpha}-\alpha) & \stackrel{d}{\rightarrow} & N\left(0, \sigma_{\alpha}\right), \\
n\left(\hat{\mu}_{\beta}-\mu_{\beta}\right) & \stackrel{d}{\rightarrow} & N\left(0, \sigma_{\beta}\right) .
\end{array}
$$

The LS estimator, the estimator $(\underline{\hat{\beta}})$ is given by $\underline{\hat{\beta}}=\left(\mathbf{X}^{\prime} \mathbf{X}\right)^{-1} \mathbf{X}^{\prime} \mathbf{Y}$. Then

$$
\hat{\hat{\beta}}=\left[\begin{array}{c}
\hat{\alpha} \\
\hat{\mu}_{\beta}
\end{array}\right]=\left[\begin{array}{cc}
n & \sum_{t=1}^{n} x_{t-1} \\
\sum_{t=1}^{n} x_{t-1} & \sum_{t=1}^{n} x_{t-1}^{2}
\end{array}\right]^{-1}\left[\begin{array}{c}
\sum_{t=1}^{n} x_{t} \\
\sum_{t=1}^{n} x_{t-1} x_{t}
\end{array}\right],
$$

following Dickey and Fuller [10], we use $(\underline{\hat{\beta}}-\underline{\beta})=\left(\mathbf{X}^{\prime} \mathbf{X}\right)^{-1} \mathbf{X}^{\prime} \underline{\varepsilon}$. So we have

$$
\begin{aligned}
{\left[\begin{array}{c}
\hat{\alpha}-\alpha \\
\hat{\mu}_{\beta}-\mu_{\beta}
\end{array}\right] } & =\left[\begin{array}{cc}
n & \sum_{t=1}^{n} x_{t-1} \\
\sum_{t=1}^{n} x_{t-1} & \sum_{t=1}^{n} x_{t-1}^{2}
\end{array}\right]^{-1}\left[\begin{array}{c}
\sum_{t=1}^{n} \varepsilon_{t} \\
\sum_{t=1}^{n} x_{t-1} \varepsilon_{t}
\end{array}\right] \\
& =\frac{1}{\delta}\left[\begin{array}{cc}
\sum_{t=1}^{n} x_{t-1}^{2} & -\sum_{t=1}^{n} x_{t-1} \\
-\sum_{t=1}^{n} x_{t-1} & n
\end{array}\right]\left[\begin{array}{c}
\sum_{t=1}^{n} \varepsilon_{t} \\
\sum_{t=1}^{n} x_{t-1} \varepsilon_{t}
\end{array}\right] \\
& =\frac{1}{\delta}\left[\begin{array}{c}
\sum_{t=1}^{n} \varepsilon_{t} \sum_{t=1}^{n} x_{t-1}^{2}-\sum_{t=1}^{n} x_{t-1} \sum_{t=1}^{n} x_{t-1} \varepsilon_{t} \\
n \sum_{t=1}^{n} x_{t-1} \varepsilon_{t}-\sum_{t=1}^{n} x_{t-1} \sum_{t=1}^{n} \varepsilon_{t}
\end{array}\right],
\end{aligned}
$$

where $\delta=n \sum_{t=1}^{n} x_{t-1}^{2}-\left(\sum_{t=1}^{n} x_{t-1}\right)^{2}$. Araveeporn [11] studied the limiting distribution using $\sqrt{n}(\hat{\theta}-\theta)$ as

$$
\begin{aligned}
n(\hat{\alpha}-\alpha) & =\frac{n \sum_{t=1}^{n} x_{t-1}^{2} \sum_{t=1}^{n} \varepsilon_{t}-n \sum_{t=1}^{n} x_{t-1} \sum_{t=1}^{n} x_{t-1} \varepsilon_{t}}{n \sum_{t=1}^{n} x_{t-1}^{2}-\left(\sum_{t=1}^{n} x_{t-1}\right)^{2}} \\
& \stackrel{d}{\rightarrow} \frac{n^{3} \sigma_{\varepsilon}^{2} \int_{0}^{1} W(r)^{2} d r W_{t}-n n^{3 / 2} \sigma_{\varepsilon} \int_{0}^{1} W(r) d r n \sigma_{\varepsilon}^{2} \int_{0}^{1} W(r) d W(r)}{\delta}
\end{aligned}
$$

where $W_{t}=\sum_{t=1}^{n} \varepsilon_{t}$. The $\mathrm{t}$ statistics of $\alpha$ can be written as

$$
t_{\alpha}=\frac{\hat{\alpha}-\alpha}{S_{\hat{\alpha}}},
$$

where

$$
S_{\hat{\alpha}}=\left\{\sigma_{\varepsilon}^{2}\left[\begin{array}{ll}
1 & 0
\end{array}\right]\left[\begin{array}{cc}
n & \sum_{t=1}^{n} x_{t-1} \\
\sum_{t=1}^{n} x_{t-1} & \sum_{t=1}^{n} x_{t-1}^{2}
\end{array}\right]^{-1}\left[\begin{array}{l}
1 \\
0
\end{array}\right]\right\}^{1 / 2}
$$


Therefore

$$
S_{\hat{\alpha}}^{2}=\sigma_{\varepsilon}^{2}\left[\frac{\sum_{t=1}^{n} x_{t-1}^{2}}{n \sum_{t=1}^{n} x_{t-1}^{2}-\left(\sum_{t=1}^{n} x_{t-1}\right)^{2}}\right],
$$

we given by

$$
n^{2} S_{\hat{\alpha}}^{2} \stackrel{d}{\rightarrow} n^{2} \sigma_{\varepsilon}^{2}\left[\frac{n^{2} \sigma_{\varepsilon}^{2} \int_{0}^{1} W(r)^{2} d r}{\delta}\right]
$$

Rewriting $t_{\alpha}$ as

$$
t_{\alpha}=\frac{n(\hat{\alpha}-\alpha)}{\left[n^{2} S_{\hat{\alpha}}^{2}\right]^{1 / 2}}
$$

and using (26) and (27), we get

$$
\begin{aligned}
t_{\alpha} & =\frac{n(\hat{\alpha}-\alpha)}{\left[n^{2} S_{\hat{\alpha}}^{2}\right]^{1 / 2}} \\
& \stackrel{d}{\rightarrow} \frac{\int_{0}^{1} W(r)^{2} d r W_{t}-n^{1 / 2} \sigma_{\varepsilon} \int_{0}^{1} W(r) d r \int_{0}^{1} W(r) d W(r)}{\left[n \sigma_{\varepsilon}^{2} \int_{0}^{1} W(r)^{2} d r\right]^{1 / 2}} .
\end{aligned}
$$

From (28), we show the limiting distribution of $\mu_{\beta}$ as

$$
\begin{aligned}
n\left(\hat{\mu}_{\beta}-\mu_{\beta}\right) & =\frac{n^{2} \sum_{t=1}^{n} x_{t-1} \varepsilon_{t}-n \sum_{t=1}^{n} x_{t-1} \sum_{t=1}^{n} \varepsilon_{t}}{n \sum_{t=1}^{n} x_{t-1}^{2}-\left(\sum_{t=1}^{n} x_{t-1}\right)^{2}} \\
& \stackrel{d}{\rightarrow} \frac{n^{2} n \sigma_{\varepsilon}^{2} \int_{0}^{1} W(r) d W(r)-n n^{3 / 2} \sigma_{\varepsilon} \int_{0}^{1} W(r) d r W_{t}}{\delta} .
\end{aligned}
$$

The $\mathrm{t}$ statistics of $\mu_{\beta}$ can be written as

$$
t_{\mu_{\beta}}=\frac{\hat{\mu}_{\beta}-\mu_{\beta}}{S_{\hat{\mu}_{\beta}}}
$$

where

$$
S_{\hat{\mu}_{\beta}}=\left\{\sigma_{\varepsilon}^{2}\left[\begin{array}{ll}
0 & 1
\end{array}\right]\left[\begin{array}{cc}
n & \sum_{t=1}^{n} x_{t-1} \\
\sum_{t=1}^{n} x_{t-1} & \sum_{t=1}^{n} x_{t-1}^{2}
\end{array}\right]^{-1}\left[\begin{array}{l}
0 \\
1
\end{array}\right]\right\}^{1 / 2} .
$$

Therefore

$$
S_{\hat{\mu}_{\beta}}^{2}=\sigma_{\varepsilon}^{2}\left[\frac{n}{n \sum_{t=1}^{n} x_{t-1}^{2}-\left(\sum_{t=1}^{n} x_{t-1}\right)^{2}}\right]
$$

we given by

$$
n^{2} S_{\hat{\mu}_{\beta}}^{2} \stackrel{d}{\rightarrow}\left[\frac{n^{3} \sigma_{\varepsilon}^{2}}{\delta}\right]
$$

Rewriting $t_{\mu_{\beta}}$ as

$$
t_{\mu_{\beta}}=\frac{n\left(\hat{\mu}_{\beta}-\mu_{\beta}\right)}{\left[n^{2} S_{\hat{\mu}_{\beta}}^{2}\right]^{1 / 2}},
$$

and using (30) and (31), we get

$$
\begin{aligned}
t_{\mu_{\beta}} & =\frac{n\left(\hat{\mu_{\beta}}-\mu_{\beta}\right)}{\left[n^{2} S_{\hat{\mu_{\beta}}}^{2}\right]^{1 / 2}} \\
& \stackrel{d}{\rightarrow} \frac{n \sigma_{\varepsilon}^{2} \int_{0}^{1} W(r) d W(r)-n^{1 / 2} \sigma_{\varepsilon} \int_{0}^{1} W(r) d r W_{t}}{\left[n \sigma_{\varepsilon}^{2}\right]^{1 / 2}} .
\end{aligned}
$$

By Slutsky's Theorem, under $t \rightarrow z$ in distribution as $n \rightarrow \infty$, where $z$ is a standard normal distribution. Therefore the $\hat{\alpha}$ and $\hat{\mu}_{\beta}$ are consistent estimator as $n \rightarrow \infty$. 


\subsection{RCA(1)-AR(1) model}

The RCA(1)-AR(1) model can be described in following simplest form

$$
x_{t}=\alpha+\mu_{\beta} x_{t-1}+\rho \varepsilon_{t-1}+e_{t}, t=1,2, \ldots, n .
$$

The RCA(1)-AR(1) from (33) can be written in terms of the matrix form as a regression model

$$
\mathbf{Y}=\mathbf{X} \underline{\beta}+\underline{e},
$$

where

$$
\mathbf{Y}=\left[\begin{array}{c}
x_{1} \\
\vdots \\
x_{n}
\end{array}\right] \quad, \quad \mathbf{X}=\left[\begin{array}{cc}
z_{0} & \hat{\varepsilon}_{0} \\
\vdots & \vdots \\
z_{n-1} & \hat{\varepsilon}_{n-1}
\end{array}\right], \quad \underline{\beta}=\left[\begin{array}{c}
v \\
\rho
\end{array}\right], \quad \underline{e}=\left[\begin{array}{c}
e_{1} \\
\vdots \\
e_{n}
\end{array}\right]
$$

and $\hat{\alpha}+\hat{\mu}_{\beta} x_{t-1}=z_{n-1}$. The limiting normal distribution is focused on the $\alpha$ and $\mu_{\beta}$, hence the Brownian motion is showed by

$$
n(\hat{\rho}-\rho) \stackrel{d}{\rightarrow} N\left(0, \sigma_{\rho}\right) .
$$

The LS estimator, the estimator $(\underline{\hat{\rho}})$ is given by $\underline{\hat{\beta}}=\left(\mathbf{X}^{\prime} \mathbf{X}\right)^{-1} \mathbf{X}^{\prime} \mathbf{Y}$. Then

$$
\begin{aligned}
\underline{\hat{\beta}} & =\left[\begin{array}{l}
\hat{v} \\
\hat{\rho}
\end{array}\right] \\
& =\left[\begin{array}{cc}
\sum_{t=1}^{n} z_{t-1}^{2} & \sum_{t=1}^{n} \hat{\varepsilon}_{t-1} z_{t-1} \\
\sum_{t=1}^{n} \hat{\varepsilon}_{t-1} z_{t-1} & \sum_{t=1}^{n} \hat{\varepsilon}_{t-1}^{2}
\end{array}\right]^{-1}\left[\begin{array}{c}
\sum_{t=1}^{n} x_{t} z_{t-1} \\
\sum_{t=1}^{n} x_{t} \hat{\varepsilon}_{t-1}
\end{array}\right],
\end{aligned}
$$

following Dickey and Fuller [10], we use $(\underline{\hat{\beta}}-\underline{\beta})=\left(\mathbf{X}^{\prime} \mathbf{X}\right)^{-1} \mathbf{X}^{\prime} \underline{e}$. Therefore we get

$$
\begin{aligned}
{\left[\begin{array}{c}
\hat{v}-v \\
\hat{\rho}-\rho
\end{array}\right] } & =\left[\begin{array}{cc}
\sum_{t=1}^{n} z_{t-1}^{2} & \sum_{t=1}^{n} \hat{\varepsilon}_{t-1} z_{t-1} \\
\sum_{t=1}^{n} \hat{\varepsilon}_{t-1} z_{t-1} & \sum_{t=1}^{n} \hat{\varepsilon}_{t-1}^{2}
\end{array}\right]^{-1}\left[\begin{array}{c}
\sum_{t=1}^{n} e_{t} z_{t-1} \\
\sum_{t=1}^{n} e_{t} \hat{\varepsilon}_{t-1}
\end{array}\right] \\
& =\frac{1}{\gamma}\left[\begin{array}{cc}
\sum_{t=1}^{n} \hat{\varepsilon}_{t-1}^{2} & -\sum_{t=1}^{n} \hat{\varepsilon}_{t-1} z_{t-1} \\
-\sum_{t=1}^{n} \hat{\varepsilon}_{t-1} z_{t-1} & \sum_{t=1}^{n} z_{t-1}^{2}
\end{array}\right]\left[\begin{array}{c}
\sum_{t=1}^{n} e_{t} z_{t-1} \\
\sum_{t=1}^{n} e_{t} \hat{\varepsilon}_{t-1}
\end{array}\right],
\end{aligned}
$$

where $\gamma=\sum_{t=1}^{n} z_{t-1}^{2} \sum_{t=1}^{n} \hat{\varepsilon}_{t-1}^{2}-\left(\sum_{t=1}^{n} \hat{\varepsilon}_{t-1} z_{t-1}\right)^{2}$. We show that

$$
\begin{aligned}
n(\hat{\rho}-\rho) & =\frac{\sum_{t=1}^{n} z_{t-1}^{2} \sum_{t=1}^{n} e_{t} \hat{\varepsilon}_{t-1}-C \sum_{t=1}^{n} e_{t} z_{t-1}}{\gamma} \\
& \stackrel{d}{\rightarrow} \frac{n^{3}\left(\sigma_{e}^{2}\right)^{2} \int_{0}^{1} W_{2}(r)^{2} d(r) \int_{0}^{1} W_{1}(r) d W_{1}(r)-C n \sigma_{e}^{2} \int_{0}^{1} W_{2}(r) d W_{2}(r)}{\gamma},
\end{aligned}
$$

where $C$ is constant. The t statistics of $\rho$ can be written as

$$
t_{\rho}=\frac{\hat{\rho}-\rho}{S_{\hat{\rho}}}
$$

where

$$
S_{\hat{\rho}}=\left\{\sigma_{e}^{2}\left[\begin{array}{ll}
0 & 1
\end{array}\right]\left[\begin{array}{cc}
\sum_{t=1}^{n} z_{t-1}^{2} & \sum_{t=1}^{n} \hat{\varepsilon}_{t-1} z_{t-1} \\
\sum_{t=1}^{n} \hat{\varepsilon}_{t-1} z_{t-1} & \sum_{t=1}^{n} \hat{\varepsilon}_{t-1}^{2}
\end{array}\right]^{-1}\left[\begin{array}{l}
0 \\
1
\end{array}\right]\right\}^{1 / 2} .
$$

Therefore

$$
S_{\hat{\rho}}^{2}=\sigma_{e}^{2}\left[\frac{\sum_{t=1}^{n} z_{t-1}^{2}}{\gamma}\right]
$$

we present that

$$
n^{2} S_{\hat{\rho}}^{2} \stackrel{d}{\rightarrow} n^{2} \sigma_{e}^{2}\left[\frac{n^{2} \sigma_{e}^{2} \int_{0}^{1} W_{2}(r)^{2} d(r)}{\gamma}\right] .
$$


Rewriting $t_{\rho}$ as

$$
t_{\rho}=\frac{n(\hat{\rho}-\rho)}{\left[n^{2} S_{\hat{\rho}}^{2}\right]^{1 / 2}}
$$

and using (38) and (39), we have

$$
\begin{aligned}
t_{\rho} & =\frac{n(\hat{\rho}-\rho)}{\left[n^{2} S_{\hat{\rho}}^{2}\right]^{1 / 2}} \\
& \stackrel{d}{\rightarrow} \frac{n^{2} \sigma_{e}^{2} \int_{0}^{1} W_{2}(r)^{2} d(r) \int_{0}^{1} W_{1}(r) d W_{1}(r)-C \int_{0}^{1} W_{2}(r) d W_{2}(r)}{\left[n^{3} \sigma_{e}^{2} \int_{0}^{1} W_{2}(r)^{2} d(r)\right]^{1 / 2}},
\end{aligned}
$$

so $\hat{\rho}$ is consistent estimator as $\alpha$ and $\mu_{\beta}$.

\section{Simulation study}

The simulation study is to compare the performance of RCA(1) model and RCA(1)-AR(1) model. We generated data from $\operatorname{RCA}(1)$ that fixed $\alpha=1$ and $\sigma_{\varepsilon}^{2}=1$ and used different values from $\left(\mu_{\beta}, \sigma_{\beta}^{2}\right)$ with parameter values as the following set;

Case $1: \mu_{\beta}=0.8$ and $\sigma_{\beta}^{2}=1$

Case $2: \mu_{\beta}=1$ and $\sigma_{\beta}^{2}=0$

Case $3: \mu_{\beta}=-0.995$ and $\sigma_{\beta}^{2}=0.1$

Case $4: \mu_{\beta}=-0.1$ and $\sigma_{\beta}^{2}=0.995$.

The Figures 1 and 2 show 100 and 500 sample sizes generated from each of the above cases. Notice Case 1 looks the stationary process, whereas in Case 2 presents nonstationary process, and Case 3 and 4 tends to oscillate around its mean.

Table 1: Average and Standard Deviation of Mean Square Errors with RCA(1) and RCA(1)-AR(1) model (sample size $n=100,500$ and 500 replications)

\begin{tabular}{c|c|c|c|c}
\hline & \multicolumn{2}{|c|}{$\mathrm{n}=100$} & \multicolumn{2}{c}{$\mathrm{n}=500$} \\
\hline Case & $\mathrm{RCA}(1)$ & $\mathrm{RCA}(1)-\mathrm{AR}(1)$ & $\mathrm{RCA}(1)$ & $\mathrm{RCA}(1)-\mathrm{AR}(1)$ \\
\hline Case 1 & $\mathbf{0 . 9 8 5}$ & 3.185 & $\mathbf{0 . 9 9 6}$ & 3.230 \\
& $(0.139)$ & $(0.489)$ & $(0.060)$ & $0.222)$ \\
\hline Case 2 & $\mathbf{0 . 9 7 0}$ & 3.983 & $\mathbf{0 . 9 9 5}$ & 3.230 \\
& $(0.136)$ & $(0.542)$ & $(0.063)$ & $(0.222)$ \\
\hline Case 3 & 61.302 & $\mathbf{0 . 2 5 8}$ & 3091.059 & $\mathbf{8 . 5 8 2}$ \\
& $(714.202)$ & $(3.053)$ & $(62224.38)$ & $170.104)$ \\
\hline Case 4 & 21.720 & $\mathbf{1 4 . 9 8 6}$ & 21.275 & $\mathbf{1 9 . 7 5 9}$ \\
& $(91.771)$ & $(42.217)$ & $(42.202)$ & $(57.966)$ \\
\hline
\end{tabular}

Table 1 provides the average and standard deviation of Mean Square Error (MSE). We also computed the Mean Square Error (MSE) defined as follows:

$$
M S E=\frac{\sum_{t=1}^{n}\left(x_{t}-\hat{x}_{t}\right)^{2}}{n}
$$

where $x_{t}$ denotes the real values and $\hat{x}_{t}$ denotes the estimated values.

It appears from Table 1 that $\mathrm{RCA}(1)$ model is well estimated in Case 1 and 2 for all sample sizes. However the RCA(1)-AR(1) model performs reasonably well in Case 3 and 4 for all sample sizes. For Case 3 and 4 , we see that the volatility is high and cluster around $\mu_{\beta}$.

\section{Application in real data}

In this section, we will applied the RCA(1), and RCA(1)-AR(1) model using the LS method that we developed in previous section. The data set, we use daily volume of the Thai gold price for selling per 1.5244 grams or called 
Case 1

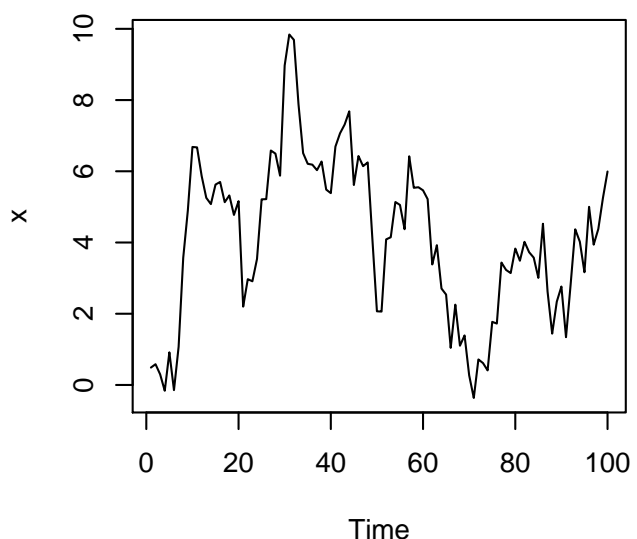

Case 3

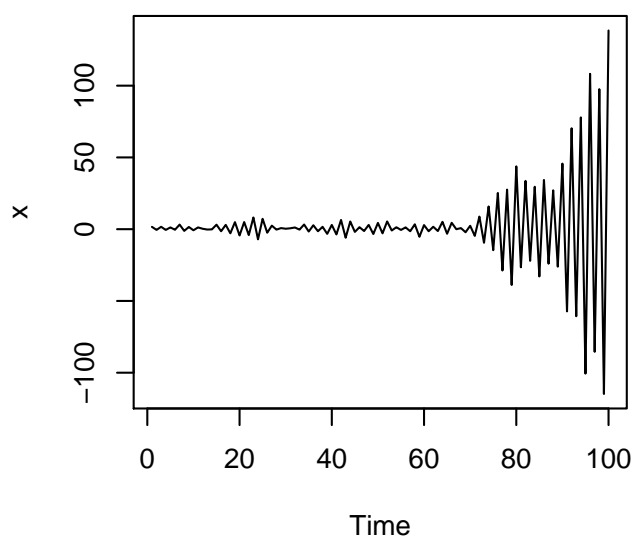

Case 2

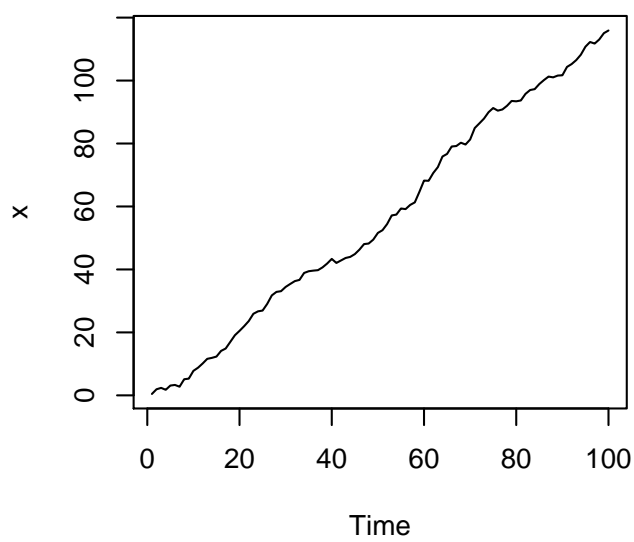

Case 4

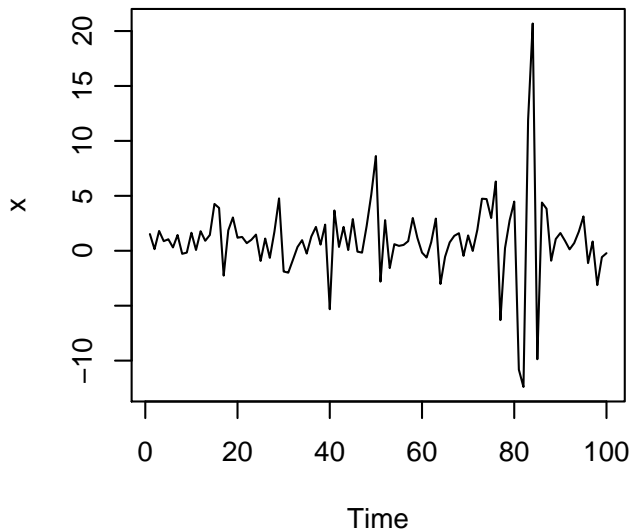

Figure 1: The time series plot for generated data (100 sample sizes)

1 Baht. This data are collected from March 1, 2012 to February 28, 2013 giving a total 288 observations which is collected from http://www.goldpricethai.com/ and shown in Figure 3.

For starters, following the RCA(1) model;

$$
\begin{gathered}
x_{t}=\alpha+\beta_{t} x_{t-1}+\varepsilon_{t}, \\
\beta_{t}=\mu_{\beta}+\sigma_{\beta} u_{t},
\end{gathered}
$$

where $\varepsilon_{t}$ 's are independently and identically distributed with mean 0 and variance $\sigma^{2}$.

Next step for parameter estimation, we fitted the RCA(1) model to obtain LS estimator $\hat{\theta}=\left(\hat{\alpha}, \hat{\mu}_{\beta}, \hat{\sigma}_{\beta}^{2}, \hat{\sigma}_{\varepsilon}^{2}\right)^{\top}$. We get

$$
\hat{\varepsilon}_{t-1}=x_{t}-\hat{\alpha}-\hat{\mu}_{\beta} x_{t-1} .
$$

Finally, the RCA(1)-AR(1) model is fitted by LS method and obtained $\hat{\theta}=\left(\hat{\alpha}, \hat{\mu}_{\beta}, \hat{\sigma}_{\beta}^{2}, \hat{\sigma}_{\varepsilon}^{2}, \hat{\sigma}_{e}^{2}, \hat{\rho}\right)^{\top}$. We have

$$
\hat{x}_{t}=\hat{\alpha}+\hat{\mu}_{\beta} x_{t-1}+\rho \hat{\varepsilon}_{t-1} .
$$

Let $x_{t}$ denote the daily volume of the Thai gold price and $\hat{x}_{t}$ denote the the daily volume of the Thai gold price estimated from RCA(1) and RCA(1)-AR(1) model. 


\section{Case 1}

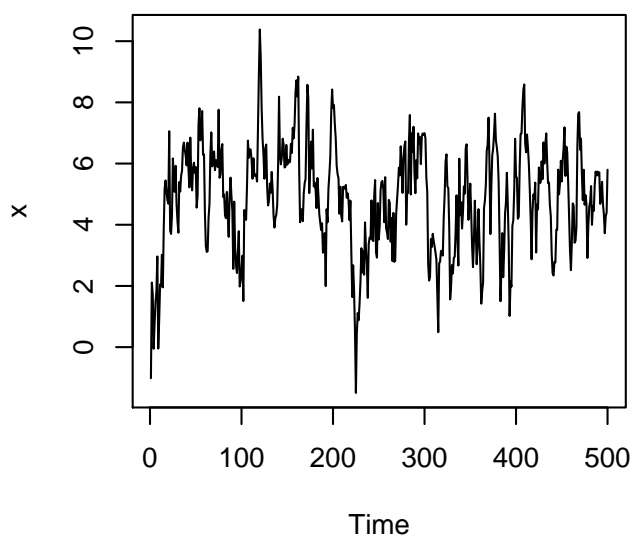

Case 3

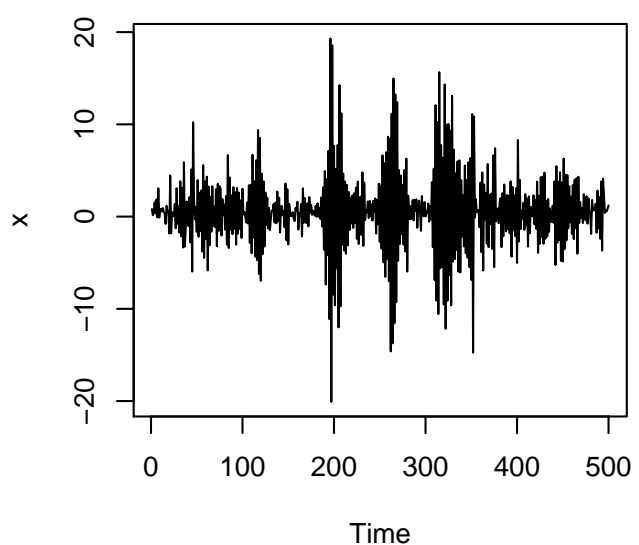

Case 2

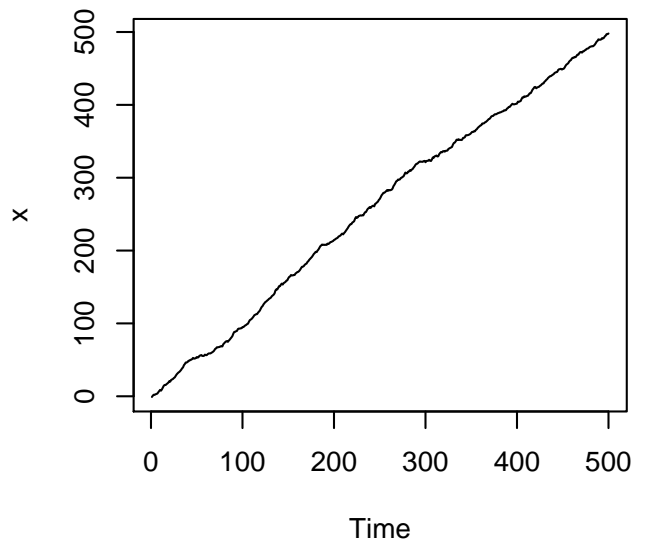

Case 4

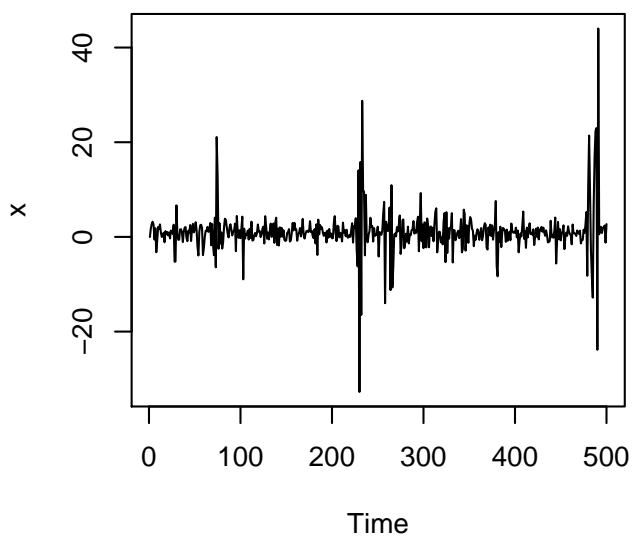

Figure 2: The time series plot for generated data (500 sample sizes)

We use the MSE to compare the performance of RCA(1) model and RCA(1)-AR(1) model. The MSE of the $\mathrm{RCA}(1)$ model (MSE $=377,077)$ is larger than the $\mathrm{RCA}(1)$ - $\mathrm{AR}(1)$ model (MSE $=1093.138)$, so the $\mathrm{RCA}(1)-\mathrm{AR}(1)$ model performs good estimates of Thai gold price.

In Figure 4 the bottom panel is the plot of Thai gold price, the dashed line is RCA(1) model, and the solid line is $\mathrm{RCA}(1)-\mathrm{AR}(1)$ model. It can be seen that the $\mathrm{RCA}(1)-\mathrm{AR}(1)$ model are competitive model when the data has volatility.

\section{Conclusion}

In this paper, we studied the LS method for RCA(1) model without correlated error and for RCA(1)-AR(1) model with autocorrelated error. We proposed to validate the efficiency of LS estimators by using Brownian motion or Wiener process that asymptotically approach the normal distribution. Through a Monte Carlo simulation study, we evaluated the performance of the LS method and showed its MSEs for different data in 4 cases at the sample size of 100 and 500. For a stationary (Case 1) and a nonstationary data (Case 2), RCA(1) model worked reasonably well for both the 100 and 500 sample size. When the data tends to oscillate around its mean (Case 3 and 4 ), the RCA(1)-AR(1) model with the LS method worked better.

For real data, we were interested in the power of estimation of each model. Using the Mean Square Error (MSE) as a criterion, we found that the RCA(1)-AR(1) model performed significantly better than the RCA(1) model. 


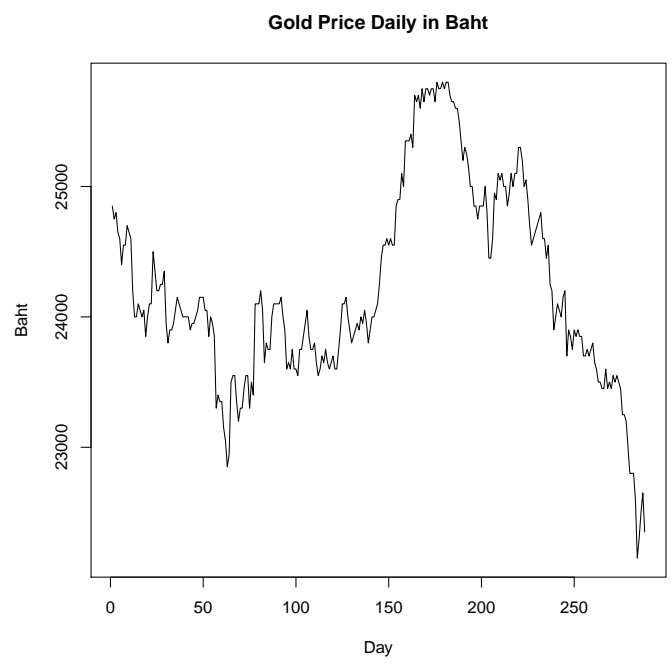

Figure 3: The time series plot of Thai gold price from March 1, 2012 to February 28, 2013

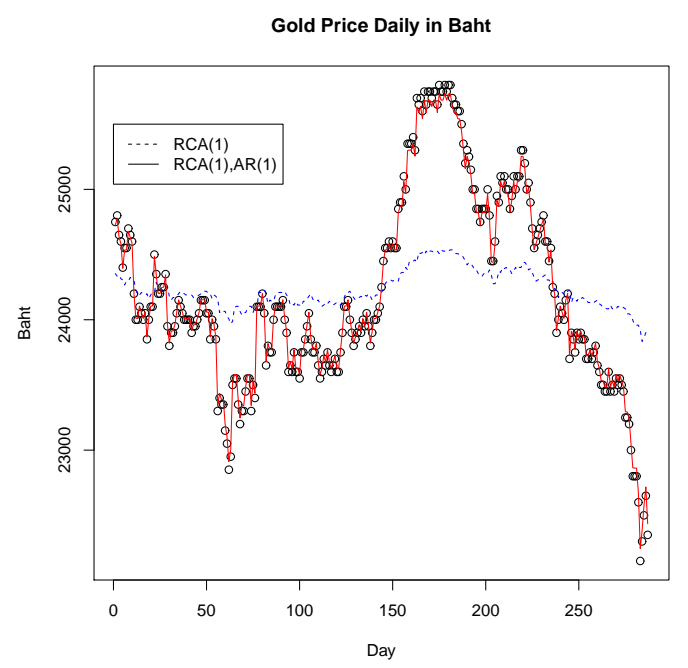

Figure 4: The scatter plot of Thai gold price and estimated parameters of $\mathrm{RCA}(1)$, and $\mathrm{RCA}(1)-\mathrm{AR}(1)$ model

As part of further work, we are going to study the following aspects of these same 2 models:

- Simultaneous estimation of the RCA-AR model using the Maximum Likelihood (ML) method approach.

- a higher order RCA model and RCA-AR model.

\section{References}

[1] D.F. Nicholls, B.G. Quinn, Random coefficient autoregressive models: An introduction, Springer- Verlag Inc (Berlin; New York), (1982).

[2] D. Wang, S.K. Ghosh, Bayesian analysis of random coefficient autoregressive models, Model Assisted Statistics and Applications, 3(2), (2002), 281-295.

[3] A. Thavaneswaran, S.S. Appadoo, S. Peiris, Forecasting Volatility, Statistics \& Probability Letters, 75, (2005), $1-10$. 
[4] S.Y. Hwang, U.V. Basawa, Parameter estimation for generalized random coefficient autoregressive process, Journal of Statistical Planning and Inference, 68, (1998), 323-337.

[5] S.A. Chandra, M. Taniguchi, Estimating Functions for Nonlinear Time Series Data, Annals of the Institute of Statistical Mathematics, 53(1), (2001), 125-136.

[6] V. Haggan, T. Ozaki, Modeling nonlinear vibrations using an amplitude-dependent autoregressive time series model, Biometrika, 68, (1981), 189-196.

[7] H. Tong, Threshold models in nonlinear time series analysis, Lecture Notes in Statistics 21, Heidelberg: Springer, (1982).

[8] K.S. Chan, H. Tong, On estimating thresholds in autoregressive models, Journal of Time Series Analysis, 7, (1986), 179-190.

[9] P.C.B. Phillips, Time series regression with a unit roots, Econometrica, 55, (1987), 227-301.

[10] D.A. Dickey, W.A. Fuller, Distribution of the estimates for autoregressive time series with a unit root, Journal of the American Statistical Association, 74, (1979), 427-431.

[11] A. Araveeporn, The Least-Squares Criteria of the Random Coefficient Dynamic Regression Model, Journal of Statistical Theory and Practice, 6(2), (2012), 315-333. 